\title{
Hepatoblast and mesenchymal cell-specific gene-expression in fetal rat liver and in cultured fetal rat liver cells
}

\author{
Tümen Mansuroglu • József Dudás · \\ Abderrahim Elmaouhoub · Tobias Z. Joza • \\ Giuliano Ramadori
}

Accepted: 26 March 2009/Published online: 19 April 2009

(c) The Author(s) 2009. This article is published with open access at Springerlink.com

\begin{abstract}
The aim of this study was to determine whether passaged rat fetal liver cells are functional hepatoblasts. Hepatocyte/hepatoblast- and liver myofibroblast-geneexpressions were studied in adult and fetal rat liver tissues as well as in primary and passaged cultures of isolated rat fetal liver cells at both the mRNA and protein level. Desmin- and Alpha-Smooth Muscle Actin (SMA)-positive cells were located in the walls of liver vessels, whereas Desmin-positive/SMA-negative cells were distributed within the liver parenchyma. Primary cultures contained Prox1-positive hepatoblasts, Desmin/SMA-positive myofibroblasts and only a few Desmin-positive/SMA-negative cells. Albumin and alpha-fetoprotein (AFP) could be detected in the primary cultures and to a lesser extent after the first passage. The number of Desmin-positive/SMA-negative cells decreased with successive passage, such that after the second passage, only Desmin/SMA-positive cells could be detected. SMAgene-expression increased during the passages, suggesting that myofibroblasts become the major cell population of fetal liver cell cultures over time. This observation needs to be taken into account, should passaged fetal liver cells be used for liver cell transplantation. Moreover it contradicts the concept of epithelial-mesenchymal transformation and
\end{abstract}

Tümen Mansuroglu and József Dudás contributed equally to this work.

T. Mansuroglu · J. Dudás · A. Elmaouhoub ·

T. Z. Joza · G. Ramadori $(\square)$

Department of Internal Medicine, Section of Gastroenterology and Endocrinology, Georg-August-University Göttingen,

Robert-Koch-Strasse 40, 37075 Göttingen, Germany

e-mail: gramado@med.uni-goettingen.de

J. Dudás

Department of Otorhinolaryngology, University Hospital

Innsbruck, Anichstrasse 35, 6020 Innsbruck, Austria suggests rather that selective overgrowth of mesenchymal cells occurs in culture.

Keywords Hepatoblasts - Mesenchymal cells . Fetal liver

\section{Introduction}

The principal functional cell type of the liver is the hepatocyte, which accounts for $78 \%$ of parenchymal volume in the rat liver. Biliary epithelial cells (cholangiocytes), sinusoidal endothelial cells, hepatic stellate cells (Ito cells), myofibroblasts, Kupffer cells, and pit cells (liver-specific natural killer cells) represent the majority of non-hepatocyte cell types in the adult liver (Zhao and Duncan 2005).

Hepatocytes and cholangiocytes derive from hepatoblasts, which emerge from the foregut endoderm and invade the septum transversum mesenchyme to form the liver bud (Duncan 2003; Shiojiri 1997; Shiojiri et al. 2001; Lemaigre and Zaret 2004). Hematopoietic cells migrate into the liver bud during embryonic day (ED) 12 in the mouse and proliferate there, apparently emitting a growth signal for the liver (Kamiya et al. 1999). The onset of synthesis of secreted proteins during rat liver development occurs around ED12, while glycogen rosettes are identifiable only after ED18 (Duncan 2003). Rat fetal hepatocytes acquire their polygonal structural characteristic just prior to birth (Vassy et al. 1988). These data suggest that hepatocytes differ functionally at different developmental times.

The liver anlage appears at ED10-12 in the rat and albumin and alpha-fetoprotein (AFP) are synthesized and secreted at this time point (Elmaouhoub et al. 2007). Synthesis and secretion of albumin and AFP can be 
detected in a few ventral foregut endodermal cells (ED1012); fibrinogen synthesis becomes detectable at ED12. These data suggest that the secretory machinery of the hepatoblasts in the liver anlage is functional from the earliest stages of liver development and that the hepatocyte secretory apparatus is already functional before the emergence of the liver bud. The ventral foregut endodermal cells manifest not only the gene-expression and protein synthesis of albumin but also the albumin processing activity before the formation of the liver anlage (Elmaouhoub et al. 2007). This observation has been confirmed by the detection of the subtilisin-like proprotein convertases, which are involved in proteolytic processing of albumin (Mori et al. 1999; Roebroek et al. 2004).

It remains unknown if subcultured fetal liver cells contain mesenchymal lineages as well and whether they expand during culture and serial passage.

This question has gained importance as several studies have reported that expanding and subcultured fetal liver cells maintain the gene-expression of some hepatocyte markers and function as hepatocytes (Oertel et al. 2006; Oertel et al. 2008).

In the current study we investigated the phenotypic and functional characteristics of fetal rat liver cells in culture. We found that after the first passage, functional hepatocellular markers such as albumin and AFP dramatically decreased and ultimately disappeared with subsequent passage. At the same time mesenchymal cells with the characteristics of myofibroblasts become the principal cell populations within the culture.

\section{Materials and methods}

Animals and preparation of embryos

Pregnant Wistar rats weighing 300-400 g were purchased from Harlan Winkelmann (Borchen, Germany). The rats were prepared on the day of delivery or kept at $19-23^{\circ} \mathrm{C}$ under standard conditions with 12-h light-dark cycles and access to food and water until the desired development stage was achieved. All animals received humane care in accordance with the institution's and the German Convention for Protection of Animals and the National
Institutes of Health guidelines. Moreover, this study was approved by the local ethics committee. The rats were anesthetized by intraperitoneal injection of pentobarbital (Narcoren, Merial, Hallbergmoos; $260 \mathrm{mg} / \mathrm{kg}$ body weight). After Narcoren anesthesia, the uterus was removed and placed in a Petri dish with cold PBS (with $\mathrm{Ca}^{++}$and $\mathrm{Mg}^{++}$).

Fetuses were obtained at different embryonic days (ED12, ED14, ED16 and ED18) and collected in ice-cold MEM Hanks with stable glutamine. The fetal liver was carefully removed and processed for primary culture, or shock frozen in liquid nitrogen at $-80^{\circ}$ until further use (immunohistochemical examination and RNA isolation).

\section{Immunohistochemical analysis}

Five-micrometer thick sections from adult rat liver and from fetal rat liver obtained at ED12, ED14, ED16, ED18 and from newborn rats were prepared with a cryostat (Reichert Jung, Wetzlar, Germany). Immunohistochemical staining with the antibodies listed in Table 1 was performed as described before (Dudas et al. 2006).

Briefly, cryostat sections were air-dried and used for immunohistochemical studies after fixation for $10 \mathrm{~min}$ in acetone at room temperature. After blocking of non-specific binding with $1 \%$ bovine serum albumin (Serva, Heidelberg, Germany) and 10\% goat serum (DAKO, Glostrup, Denmark) contained in PBS (Biochrom, Berlin, Germany) for $1 \mathrm{~h}$ at room temperature, two primary antibodies were simultaneously incubated overnight at $4^{\circ} \mathrm{C}$ on the sections (1:50; DAKO, Glostrup, Denmark). Double immunostaining for studies of antigen co-localization was performed using the fluorescent conjugates. The rabbit polyclonal and mouse monoclonal antibodies were detected with Alexa-555-conjugated goat-anti-rabbit and Alexa488 conjugated goat-anti-mouse secondary antibodies (Molecular Probes, Leiden, Netherlands) diluted 1:400 and 1:200 in PBS. Sections were counter-stained with DAPI (Molecular Probes) and analyzed with an epifluorescence microscope (Axiovert 200M with Apotome function; Zeiss, Jena, Germany). Microphotographs were obtained using Axiovision 4.2 and 4.5 Zeiss software. Negative control immunostainings were performed by omission of

Table 1 Antibodies used in the study

\begin{tabular}{lllll}
\hline Antibody & Source & Usage & Dilution & Provider \\
\hline Anti-Prox1 & Rabbit polyclonal & Double staining with Desmin or with SMA & $1: 500$ & Reliatech (Braunschweig, Germany) \\
Anti-SMA & Mouse monoclonal & Double staining with Prox1 & $1: 250$ & Sigma (Steinheim, Germany) \\
Anti-SMA & Rabbit polyclonal & Double staining with Desmin & $1: 500$ & Epitomics (Hamburg, Germany) \\
Anti-Desmin & Mouse monoclonal & Double staining with Prox1 & $1: 50$ & DAKO (Glostrup Denmark) \\
\hline
\end{tabular}


the primary antibody, using isotype-matching control immunoglobulins and using antibodies directed against antigens not expressed in rat liver.

Isolation of primary fetal liver cells

After preliminary experiments evaluating hepatoblast recovery at time points ED12, ED14, ED16 and ED18, the highest reproducible rates of cell yield were identified at ED18. These cells obtained at ED18 were studied in detail. The fetal liver was dissected using a binocular microscope and incubated in MEM Hanks medium with $25 \mathrm{mM}$ HEPES, stable glutamine-supplemented with $0.05 \%$ DNase and $0.05 \%$ collagenase $\mathrm{H}$ as previously described (Elmaouhoub et al. 2007). The isolated cells were suspended in William's E medium (Biochrom, Berlin, Germany) supplemented with $10 \%$ fetal calf serum, $100 \mathrm{U} / \mathrm{ml}$ penicillin, $100 \mu \mathrm{g} / \mathrm{ml}$ streptomycin, $1 \%$ L-glutamine, $0.1 \mu \mathrm{g} / \mathrm{ml}$ insulin, $0.1 \mu \mathrm{g} / \mathrm{ml}$ glucagon and $0.1 \mu \mathrm{g} / \mathrm{ml}$ dexamethasone. Cells were seeded onto collagen type I-coated 24-well plates (BD, Bedford, MA, USA) and 8-chambered slides (Nunc, Roskilde, Denmark) and incubated at $37^{\circ} \mathrm{C}$ in a humidified $5 \% \mathrm{CO}_{2}$ incubator. After $24 \mathrm{~h}$, the non-adherent cells were collected and removed. The adherent cells were washed twice with PBS. Adherent and non-adherent cells were cultured for an additional $24 \mathrm{~h}$ in serum-free hybridomed medium (DIF) supplemented with $100 \mathrm{U} / \mathrm{ml}$ penicillin, $100 \mu \mathrm{g} / \mathrm{ml}$ streptomycin, $1 \% \mathrm{~L}$-glutamine, $0.1 \mu \mathrm{g} / \mathrm{ml}$ insulin, $0.1 \mu \mathrm{g} / \mathrm{ml}$ glucagon and $0.1 \mu \mathrm{g} / \mathrm{ml}$ dexamethasone.

Both adherent and non-adherent cells were analyzed as primary cultures $48 \mathrm{~h}$ after plating. The adherent fetal liver cells were recovered after trypsinizing and plated onto collagen type I-coated 24-well plates and were cultured in serum free hybridomed medium (DIF). Special care was taken to provide identical culture conditions for both the primary and passaged cells. Cell passage was repeated twice (P0: primary culture; $\mathrm{P} 1$ : first passage; $\mathrm{P} 2$ : second passage; P3: third passage). Both the primary cultures and the passaged cultures were subconfluent when studied.

Immunocytochemical analysis of primary and passaged fetal liver cell cultures

For the immunofluorescence studies, primary or passaged adherent liver cells were cultured for 2 days on collagencoated 8-chambered permanox slides, fixed in acetone for $10 \mathrm{~min}$ at room temperature and rinsed in phosphate-buffered saline. Primary non-adherent liver cells were prepared as cytospin smears after $48 \mathrm{~h}$ of primary culture. The viability of the non-adherent cells was examined and was found to be greater than $85 \%$. The cytospin smears were fixed and washed identical to the adherent culture in 8-chambered slides. Blocking of non-specific binding was performed with $1 \%(\mathrm{w} / \mathrm{v})$ bovine serum albumin and $10 \%$ $(\mathrm{v} / \mathrm{v})$ goat serum (DAKO) in PBS for $1 \mathrm{~h}$ at RT. The slides were then immunostained with the antibodies listed in Table 1 . The rabbit polyclonal antibodies were detected with Alexa 555-conjugated secondary anti-rabbit antibody, mouse monoclonal antibodies with Alexa 488-conjugated secondary anti-mouse antibody (Molecular Probes,) diluted 1:400 and 1:200 in PBS, respectively. The slides were counter-stained with 4'-6-diamidino-2-phenylindole (DAPI) (Molecular Probes) and were analyzed with an epifluorescence microscope (Axioskop 50 or Axiovert 200M, Zeiss).

Isolation of total RNA and real-time RT-PCR analysis

Total RNA was isolated from cultured cells and fetal liver tissue by means of guanidine isothiocyanate extraction, cesium chloride density-gradient ultracentrifugation and ethanol as described elsewhere (Elmaouhoub et al. 2007). cDNAs were created by using M-MLV Reverse Transcriptase (RT) Kit (Invitrogen, Carlsbad, CA, USA). cDNAs from cultured primary and passaged liver cells as well as from fetal liver tissue were prepared using the Fastlane Cell cDNA kit (Qiagen, Hilden, Germany).

mRNA expression was measured quantitatively during liver development by real-time RT-PCR using an ABI Prism 7000 thermal cycler (Applied Biosystems, Darmstadt, Germany), SYBR ${ }^{\circledR}$ Green PCR Mastermix (Invitrogen), cDNA and specific primers described in previous studies: albumin, AFP, Ubiquitin C (UBC); SMA, and Desmin (Elmaouhoub et al. 2007; Dudas et al. 2009, 2006). The gene-expression levels of target genes $(Q)$ were determined based on the threshold PCR cycle-values $\left(C_{\mathrm{t}_{\text {target }}}\right)$ (following the instructions of Applied Biosystems) and were normalized with the threshold PCR cycle-values of the endogenous control UBC $\left(C_{\mathrm{t}_{185}}\right)$, using the following formula: $Q=2^{-\Delta C_{\mathrm{t}}}$, where $\Delta C_{\mathrm{t}}=C_{\mathrm{t}_{\mathrm{target}}}-C_{t_{185}}$.

Biosynthetic radioactive labeling, immunoprecipitation and SDS-PAGE of newly synthesized proteins

Newly synthesized proteins were radioactively labeled with ${ }^{35} \mathrm{~S}$-methionine as previously described (Ramadori et al. 1985; Elmaouhoub et al. 2007).

Briefly, endodermal cells derived from the ventral foregut, hepatoblasts and adherent passaged cells at different developmental stages were cultured for 2 days and then washed three times with methionine-free RPMI medium and labeled in RPMI medium supplemented with ${ }^{35} \mathrm{~S}$-methionine $(100 \mu \mathrm{Ci} /$ well $)$ for $2 \mathrm{~h}$. Cells derived 
from the ventral foregut were labeled overnight. After labeling, the supernatants were harvested and diluted to $50 \%$ with lysis buffer with sodium dodecylsulfate $(0.5 \%$ (w/v) (deoxycholic acid (DOC), 1\% (v/v) Triton X-100, $10 \mathrm{mM}$ ethylene-diaminetetraacetic acid (EDTA), $1 \%(\mathrm{w} / \mathrm{v})$ sodium dodecylsulfate contained in PBS. $\mathrm{pH}$ 7.4). The cells were lysed after freeze-thawing and scraping in lysis buffer without sodium dodecylsulfate supplemented with $1 \% \quad(\mathrm{v} / \mathrm{v})$ phenylmethylsulfonyl fluoride $(2 \mathrm{mM}$ PMSF in ethanol, $\mathrm{pH}$ 7.4). Cell-lysates were then harvested and diluted to $50 \%$ with lysis buffer with sodium dodecylsulfate. The radioactivity of the total labeled proteins was determined after a trichloroacetic acid precipitation.

For immunoprecipitation of albumin and AFP (Dako, Glostrup, Denmark), the supernatants and cell-lysates were used and incubated with specific antibodies and protein precipitation was achieved by using protein A (Ramadori et al. 1987). The immunoprecipitated albumin and AFP were analyzed using 12\% SDS-polyacrylamide gel electrophoresis (SDS-PAGE) and the radioactivity in the labeled proteins was identified by autoradiography (Elmaouhoub et al. 2007).
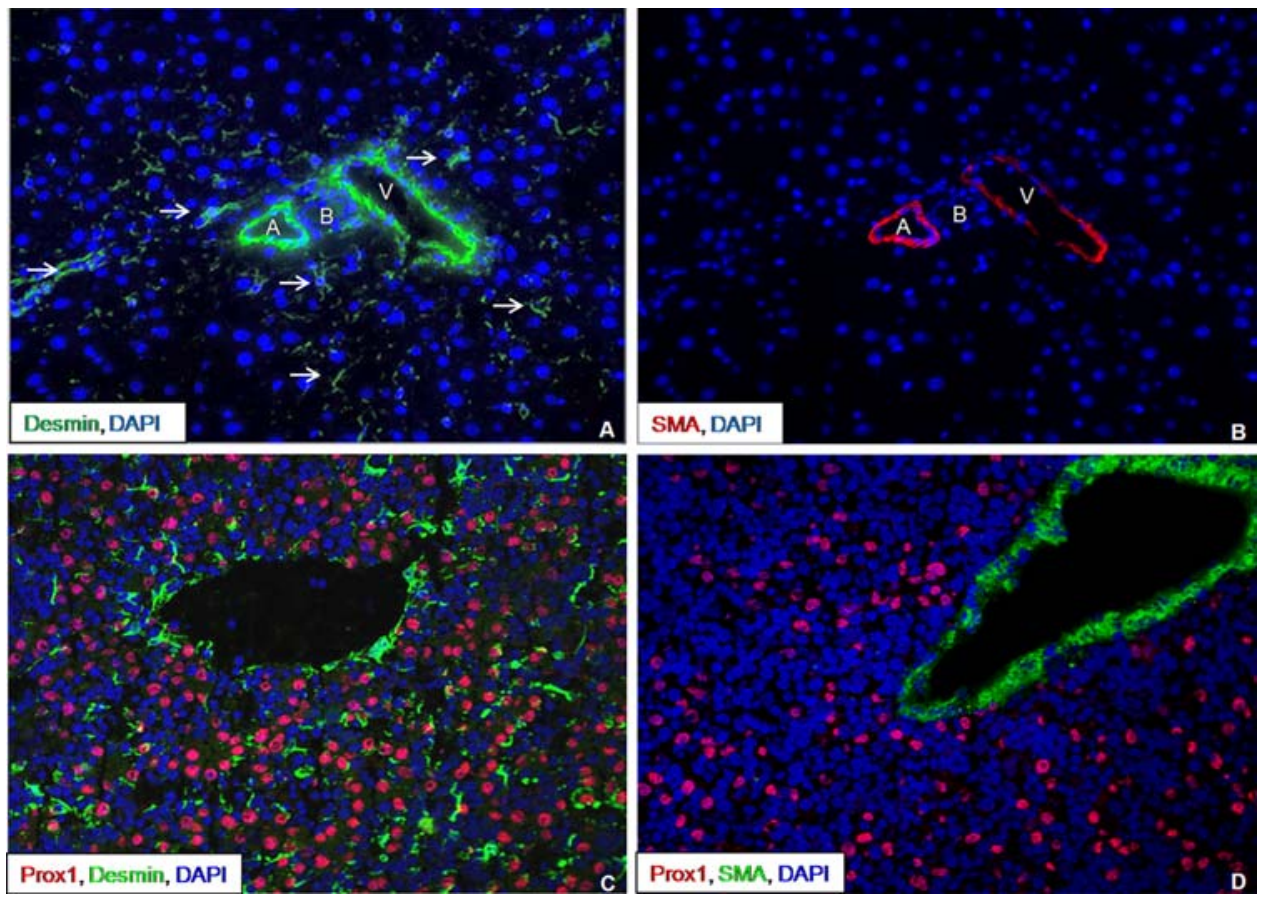

Fig. 1 Double-immunofluorescence staining of Desmin (detected in green) and SMA (detected in red) in adult normal rat liver tissue illustrated separately (a, b). Desmin and SMA positivity could be detected in the wall of the hepatic artery (A) and portal vein $(\mathrm{V})$, but not in the bile duct (B). Note that Desmin-positive cells could be detected within the sinusoids of the liver parenchyma as well (arrows). Double-immunofluorescence staining of Prox1 (detected in magnification)

\section{Results}

Immunohistochemical expression of hepatoblast and mesenchymal cell marker genes in adult and fetal rat liver

Adult rat liver showed SMA- and Desmin-positivity by cells of vessel walls (Fig. 1a, b), and Desmin-alone within the liver sinusoids (Fig. 1a). Hepatocyte nuclei were Prox 1 positive (Dudas et al. 2004, 2006). The same pattern of positivity was observable in fetal liver hepatocytes (Fig. 1c, d). At ED14-18, the majority of Prox1-positive cells were also AFP-positive (Dudas et al. 2006).

Immunocytochemical detection of hepatoblast and mesenchymal cell markers in cultured fetal liver cells

Cultures of rat fetal liver cells contained epithelial cells, representing Prox1-positive hepatoblasts which formed aggregates or clusters (Fig. 2a, b). Additionally, Desminand SMA-positive cells were detected at the border of the Prox1-positive hepatoblast clusters (labeled with "M";

red) and Desmin or SMA (detected in green) in rat fetal liver tissue (ED 18) revealed Desmin-positive cells in the wall of the liver vessel and within the sinusoids of the liver parenchyma (c). SMA-positive cells could only be detected in the wall of the liver vessel (D). Note that the hepatocytes were consistently negative for Desmin and SMA. The blue staining with DAPI represents the nuclei $(200 \times$ original 


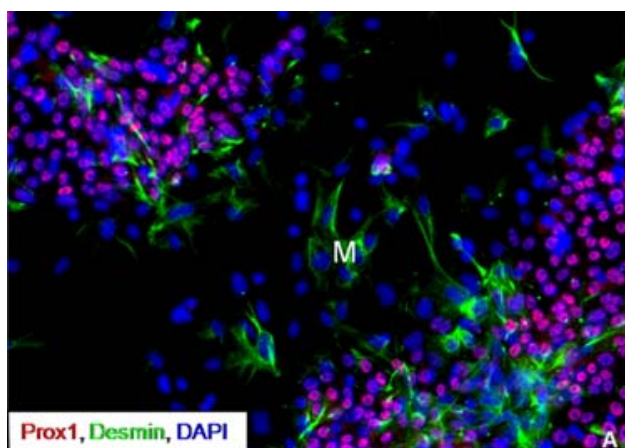

Fig. 2 Double-immunocytochemical staining of Prox 1 (detected in red) with Desmin and SMA (detected in green) in primary culture of adherent rat fetal liver cells. The blue staining with DAPI represents the nuclei (a $200 \times$ original magnification; b $100 \times$ original magnification). The primary adherent culture of rat fetal liver cells

Fig. 2a, b). Compared to Desmin- and SMA-positive cells, hepatoblasts exhibited a cuboidal shape. Prox1-positive hepatoblasts were consistently negative for Desmin and SMA staining.

The same markers were investigated in the passaged cultures of rat fetal liver cells, having a spindle-like morphology and manifesting a Desmin- and/or SMA-positive staining, but no Prox1 expression. This finding suggested that two different populations of mesenchymal cells were present in rat fetal liver (Fig. 3). Double-immunocytochemical labeling of Desmin with SMA revealed Desmin-negative/SMA-positive cells as well as Desmin- and SMA-positive cells in the passaged cultures (Fig. 4).

Expression of hepatocellular and mesenchymal marker genes in fetal liver and in primary and passaged fetal liver cells

Albumin and AFP were highly expressed in the rat fetal liver tissue at ED18. The primary adherent liver cell cultures had a comparable albumin-gene-expression level to that of the fetal liver tissue (Fig. 5a). The non-adherent fetal rat liver cells expressed albumin and AFP only minimally (100-fold lower level compared to the adherent culture; Fig. 5a, b). The gene-expression pattern of albumin and AFP was lost in subsequently passaged cells (third passage, Fig. 5a, b).

In fetal liver tissue, the gene-expression level of Desmin and SMA was significantly less than the expression of albumin and AFP $(p<0.01$; Fig. 5c, d). Additionally, the gene-expression of SMA and Desmin was measured in fetal liver tissues as well as in primary cultures of adherent fetal liver cells, obtaining a higher mRNA expression of SMA and Desmin in the cultured cells (Fig. 5c, d). SMA could not be detected in the primary culture of nonadherent fetal liver cells, while Desmin showed a significantly lower expression as compared with the adherent

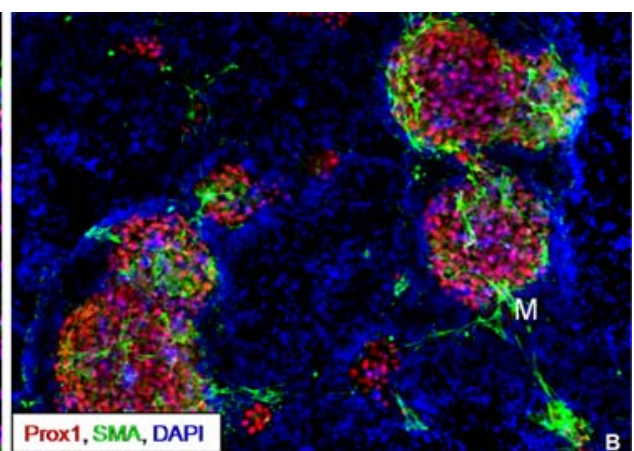

contained epithelial cell aggregates representing Prox1-positive hepatoblasts and Desmin- and SMA-positive mesenchymal cells (labeled with M). No Desmin- and SMA positivity could be detected in Prox1-positive hepatoblasts

cells $(p<0.05$; Fig. 5a). The gene-expression of these mesenchymal markers was analyzed in passaged fetal liver cells and SMA-gene-expression increased, while the geneexpression level of Desmin decreased compared with that of the primary cultures ( $p<0.05$; Fig. 5a-c), suggesting a selection of SMA-positive mesenchymal cells with successive passage of isolated fetal liver cells.

Albumin and AFP synthesis and secretion in primary and passaged fetal liver cell cultures

A sensitive radioactive biosynthetic labeling and immunoprecipitation method was used to assess synthesis and secretion of albumin and AFP in primary fetal liver cell cultures, and after successive passage of the cells. High synthesis and secretion levels of albumin and AFP by primary adherent rat fetal liver cell cultures were observed (Fig. 6a, b). No albumin synthesis and secretion was detected in the cell lysate and supernatant of non-adherent rat fetal liver cell cultures (Fig. 6a, b). After the first passage, albumin and AFP synthesis and secretion was still detectable, but was significantly reduced (Fig. 6b). After the second passage albumin and AFP synthesis and secretion was not detectable (Fig. 6b), indicating a loss of hepatoblasts during the passaging procedure.

\section{Discussion}

In the current study, vascular walls in the rat fetal liver tissue contain mesenchymal cells which are positive for both Desmin and SMA and are negative for Prox 1, an early marker of hepatoblasts (Dudas et al. 2004). Mesenchymal cells of the fetal liver parenchyma are Desmin-positive. The detectability of Desmin-positive cells in the parenchyma of fetal liver is a result of the incomplete/absence of hepatocyte plates and sinusoids in the fetal liver. 


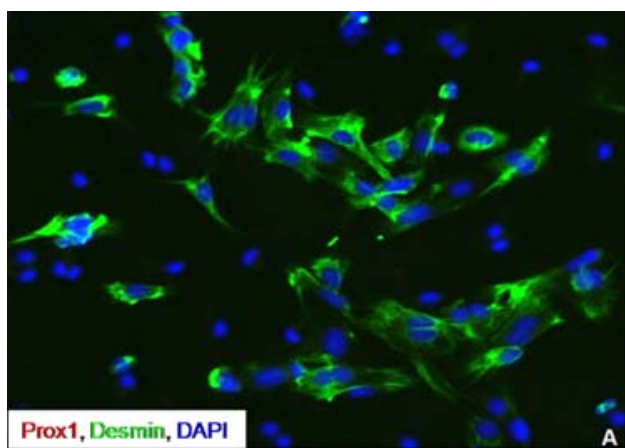

Fig. 3 Double-immunocytochemical labeling of Desmin and SMA (detected in green) with Prox1 (detected in red) in passaged (second passage) cultures of rat fetal liver cells. Negative control was performed using an isotype-matching primary immunoglobulin. The

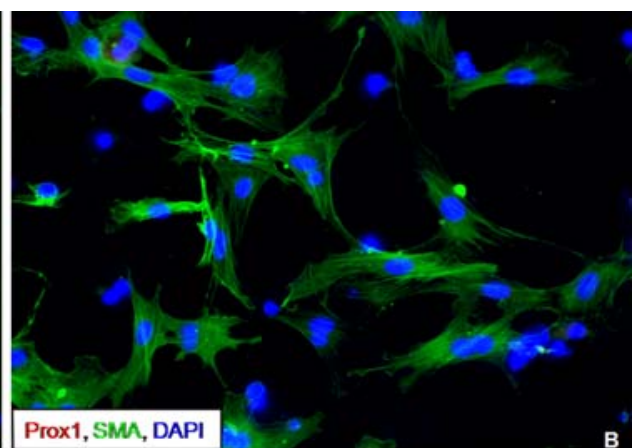

blue staining with DAPI represents the nuclei $(200 \times$ original magnification). Note that passaged cultures contained Desmin- and SMA-positive cells, whereas no Prox1-positivity could be detected in the nuclei
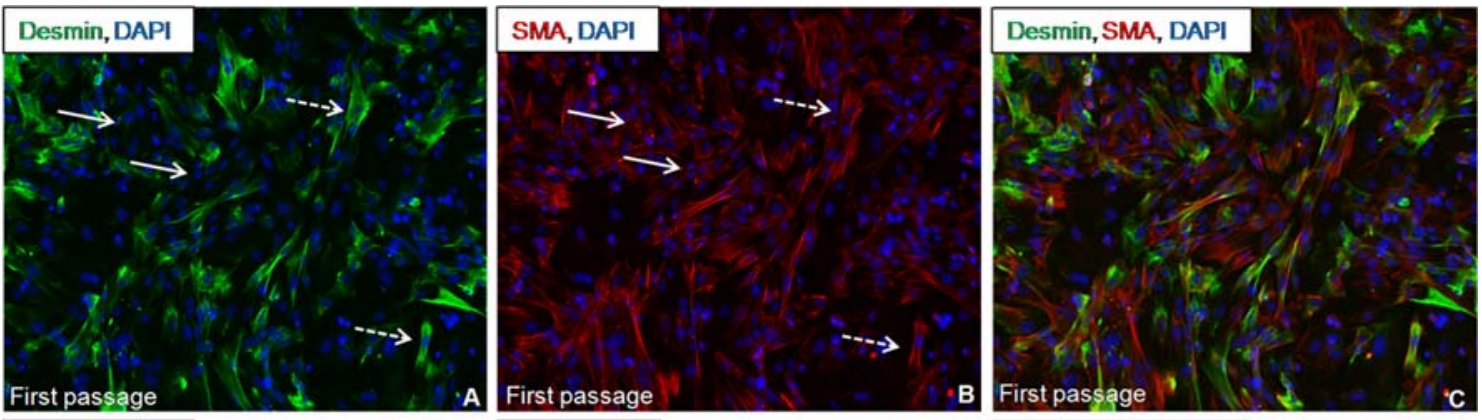
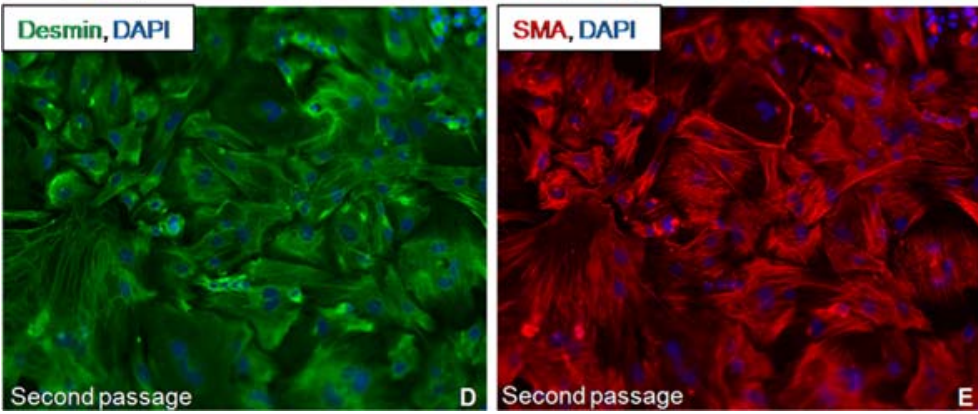

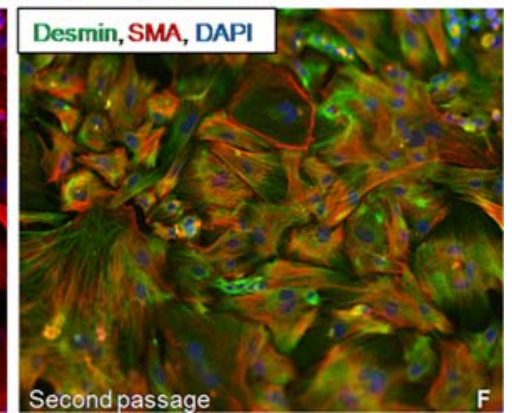

Fig. 4 Double-immunocytochemical labeling of Desmin (detected in green) and SMA (detected in red) in passaged cultures of rat fetal liver cells, illustrated separately $(\mathbf{a}, \mathbf{b} ; \mathbf{d}, \mathbf{e})$ and merged $(\mathbf{c}, \mathbf{f})$. The blue staining with DAPI represents the nuclei $(\mathbf{a}-\mathbf{c} 100 \times$, d-f $200 \times$ original magnification). Note that Desmin-negative/SMA-positive cells (continuous arrows) as well as Desmin- and SMA-positive cells (dashed arrow) could be detected after the first passage (a-c). After the second passage only Desmin- and SMA-positive cells were detectable $(\mathbf{d}-\mathbf{f})$

passage, a dramatic loss of hepatoblasts occurs and the expression of albumin and AFP is reduced after the first passage and is completely absent after the second passage.

The decreased expression of classical hepatocyte/ hepatoblast cellular function, namely synthesis of albumin and AFP, found in passaged rat fetal liver cells was confirmed, utilizing a very specific and sensitive method of the radioactive biosynthetic protein labeling and immunoprecipitation followed by SDS-PAGE analysis and autoradiography of the immunoprecipitates.

In the primary and passaged cultures of adherent fetal liver cells, SMA expression was greater than that found in 
Fig. 5 Comparison of the geneexpression of hepatocyte/ hepatoblast $(\mathbf{a}, \mathbf{b})$ and of liver mesenchymal markers $(\mathbf{c}, \mathbf{d})$ in rat fetal liver tissue, in primary culture of adherent (designated as "Primary cult") and nonadherent fetal liver cells as well as in passaged fetal liver cells
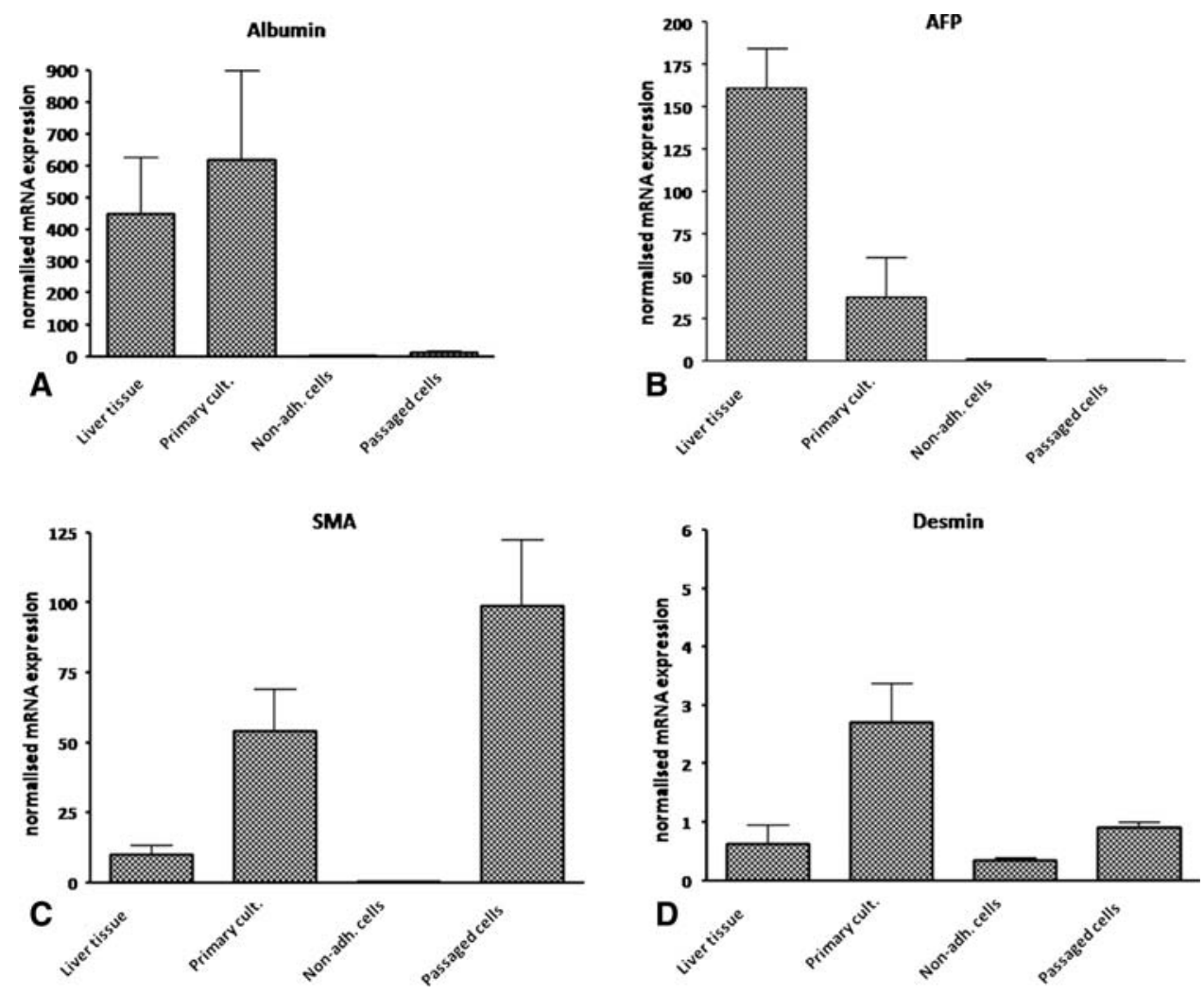

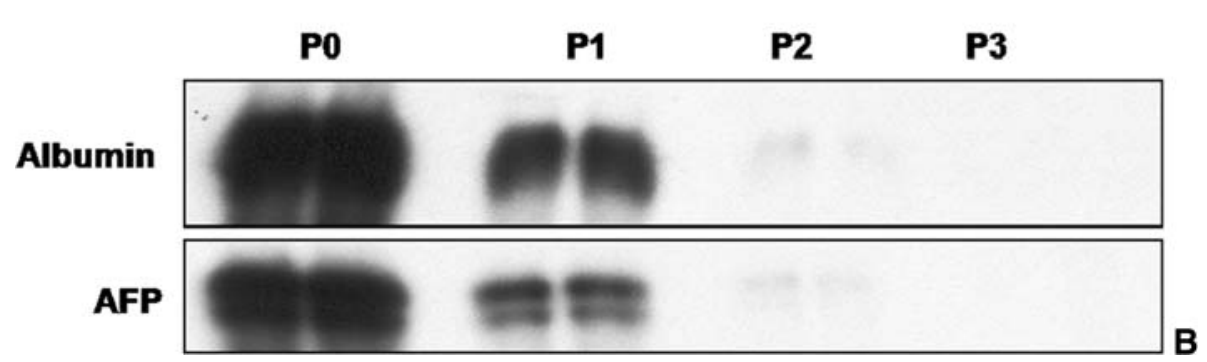

Fig. 6 a Utilization of the radioactive biosynthetic labeling and immunoprecipitation to assess synthesis and secretion of albumin in primary adherent and non-adherent as well as in passaged cultures of rat fetal liver cells ( $L$ cell-lysat, $S$ supernatant, $P$ passage number). b Utilization of the radioactive biosynthetic labeling and immunoprecipitation to assess synthesis and secretion of albumin and AFP in primary and passaged liver cell cultures (P1, P2 and P3). Each sample

fetal liver tissue. In contrast to SMA, the gene-expression of Desmin increased in primary cultures and was reduced in passaged cultures.

The current study demonstrates that the major component of passaged fetal liver cells consists of a Desmin- and represented in duplicate, showing two separate isolations of the cells. Albumin and AFP were immunoprecipitated with polyclonal antialbumin and anti-AFP antibodies. The immunocomplexes were analyzed by SDS-PAGE. Cell-lysates (intracellular) and supernatants (extracellular) were used for immunoprecipitation by taking into consideration samples with similar count

SMA-positive cell population characteristic of myofibroblasts within adult rat liver.

The presence of mesenchymal cells in developing rat and mouse fetal livers has been analyzed earlier (Vassy et al. 1993; Nitou et al. 2000). 
Nitou et al. demonstrated Desmin-positive cells at early stages of mouse liver development. These cells differed from hepatoblasts and hepatocytes in terms of their morphology. Fetal hepatoblasts and hepatocytes did not react with anti-Desmin antibodies, nor did Desmin-positive cells express cytokeratins and E-cadherin, which were used as a marker for hepatoblasts (Nitou et al. 2000).

Dan et al. reported the isolation and characterization of a stable population of human liver progenitor cells from human fetal liver that does not express liver-specific genes, but is able to differentiate into functional hepatocytes and bile duct cells (Dan et al. 2006). Moreover, these human fetal liver multipotent progenitor cells showed features of mesenchymal-epithelial transition; retained multipotent capability to differentiate into fat, cartilage, bone, and endothelial cells; and were reported to have a repopulation capacity in a mouse model of liver injury. When grown on collagen plates, human fetal liver multipotent progenitor cells flattened into a monolayer, progressively increased in size, and assumed a polygonal morphology which was thought to resemble that of hepatocytes. AFP expression appeared only transiently and no albumin could be identified in the culture medium.

In 1995, Pagan et al. reported that hepatocytes from either neonatal or adult rat liver, if maintained in monolayer culture for several weeks, are replaced by cells that bear no phenotypic resemblance to hepatocytes and explained the appearance of these cells as a result of epithelial-mesenchymal transition (Pagan et al. 1995).

In contrast, in a study by Deurholt et al. human fetal liver cells (HFLCs) were isolated from fetal livers at 16 weeks of gestation. The hepatic function of these HFLCs was assessed in primary culture and after expansion in vitro. No differences were observed between primary HFLCs and mature human hepatocytes in terms of albumin production and the mRNA levels of various liver-specific genes. Expanding HFLCs showed decreased hepatic functions and increased cell stretching, which was similar to the observation of Douarin et al. in 1975 (Deurholt et al. 2006; Douarin 1975). Moreover, these HFLCs could not replace mature human hepatocytes in in vitro applications requiring in vitro expansion (Deurholt et al. 2006).

What the ideal culture conditions are in order to maintain hepatoblasts in culture is not known. Recent studies have indicated that hepatocyte growth factor (HGF), epidermal growth factor (EGF) and several additional factors are required to maintain functional hepatoblasts in culture (Nitou et al. 2002; Strick-Marchand and Weiss 2003; Suzuki et al. 2003; Tomizawa et al. 2008; Miki et al. 2008; Cheng et al. 2008). The characteristics of these cultured hepatoblasts have to be well defined as some proteins expressed by hepatoblasts are also expressed by other cells.
In conclusion, the current study indicates that passaged rat fetal liver cells are not functional hepatoblasts as the expression of hepatocyte markers disappeared, and the synthesis and secretory function of hepatocytes was lost. At the same time SMA- and Desmin-positive mesenchymal cells are selected. These cells most probably arise from the mesenchymal cell populations of fetal liver vascular walls and are not a result of epithelial-mesenchymal transition. Obviously, these cells cannot be used to replace hepatocytes in acutely damaged livers.

Acknowledgments We are grateful to Ms. Ch. Hoffmann, Ms. A. Herbst and Ms. S. Georgi for their excellent technical assistance. This work was supported by grants from the Deutsche Forschungsgemeinschaft (SFB 402, projects C6, D3, D4).

Open Access This article is distributed under the terms of the Creative Commons Attribution Noncommercial License which permits any noncommercial use, distribution, and reproduction in any medium, provided the original author(s) and source are credited.

\section{References}

Cheng N, Wauthier E, Reid LM (2008) Mature human hepatocytes from ex vivo differentiation of alginate-encapsulated hepatoblasts. Tissue Eng Part A 14:1-7

Dan YY, Riehle KJ, Lazaro C, Teoh N, Haque J, Campbell JS, Fausto $\mathrm{N}$ (2006) Isolation of multipotent progenitor cells from human fetal liver capable of differentiating into liver and mesenchymal lineages. Proc Natl Acad Sci USA 103:9912-9917

Deurholt T, ten BL, Chhatta AA, van Wijk AC, Weijer K, Seppen J, Elferink RP, Chamuleau RA, Hoekstra R (2006) In vitro functionality of human fetal liver cells and clonal derivatives under proliferative conditions. Cell Transplant 15:811-822

Douarin NM (1975) An experimental analysis of liver development. Med Biol 53:427-455

Dudas J, Papoutsi M, Hecht M, Elmaouhoub A, Saile B, Christ B, Tomarev SI, von Kaisenberg CS, Schweigerer L, Ramadori G, Wilting J (2004) The homeobox transcription factor Prox 1 is highly conserved in embryonic hepatoblasts and in adult and transformed hepatocytes, but is absent from bile duct epithelium. Anat Embryol (Berl) 208:359-366

Dudas J, Elmaouhoub A, Mansuroglu T, Batusic D, Tron K, Saile B, Papoutsi M, Pieler T, Wilting J, Ramadori G (2006) Prosperorelated homeobox 1 (Prox 1 ) is a stable hepatocyte marker during liver development, injury and regeneration, and is absent from "oval cells". Histochem Cell Biol 126:549-562

Dudas J, Mansuroglu T, Batusic D, Ramadori G (2009) Thy-1 is expressed in myofibroblasts but not found in hepatic stellate cells following liver injury. Histochem Cell Biol 131:115-127

Duncan SA (2003) Mechanisms controlling early development of the liver. Mech Dev 120:19-33

Elmaouhoub A, Dudas J, Ramadori G (2007) Kinetics of albuminand alpha-fetoprotein-production during rat liver development. Histochem Cell Biol 128:431-443

Kamiya A, Kinoshita T, Ito Y, Matsui T, Morikawa Y, Senba E, Nakashima K, Taga T, Yoshida K, Kishimoto T, Miyajima A (1999) Fetal liver development requires a paracrine action of oncostatin $M$ through the gp130 signal transducer. EMBO J $18: 2127-2136$ 
Lemaigre F, Zaret KS (2004) Liver development update: new embryo models, cell lineage control, and morphogenesis. Curr Opin Genet Dev 14:582-590

Miki R, Tatsumi N, Matsumoto K, Yokouchi Y (2008) New primary culture systems to study the differentiation and proliferation of mouse fetal hepatoblasts. Am J Physiol Gastrointest Liver Physiol 294:G529-G539

Mori K, Imamaki A, Nagata K, Yonetomi Y, Kiyokage-Yoshimoto R, Martin TJ, Gillespie MT, Nagahama M, Tsuji A, Matsuda Y (1999) Subtilisin-like proprotein convertases, PACE4 and PC8, as well as furin, are endogenous proalbumin convertases in HepG2 cells. J Biochem 125:627-633

Nitou M, Ishikawa K, Shiojiri N (2000) Immunohistochemical analysis of development of desmin-positive hepatic stellate cells in mouse liver. J Anat 197(Pt 4):635-646

Nitou M, Sugiyama Y, Ishikawa K, Shiojiri N (2002) Purification of fetal mouse hepatoblasts by magnetic beads coated with monoclonal anti-E-cadherin antibodies and their in vitro culture. Exp Cell Res 279:330-343

Oertel M, Menthena A, Dabeva MD, Shafritz DA (2006) Cell competition leads to a high level of normal liver reconstitution by transplanted fetal liver stem/progenitor cells. Gastroenterology 130:507-520

Oertel M, Menthena A, Chen YQ, Teisner B, Jensen CH, Shafritz DA (2008) Purification of fetal liver stem/progenitor cells containing all the repopulation potential for normal adult rat liver. Gastroenterology 134:823-832

Pagan R, Llobera M, Vilaro S (1995) Epithelial-mesenchymal transition in cultured neonatal hepatocytes. Hepatology 21:820-831

Ramadori G, Sipe JD, Dinarello CA, Mizel SB, Colten HR (1985) Pretranslational modulation of acute phase hepatic protein synthesis by murine recombinant interleukin 1 (IL-1) and purified human IL-1. J Exp Med 162:930-942
Ramadori G, Rieder H, Knittel T, Dienes HP, Meyer zum Buschenfelde KH (1987) Fat storing cells (FSC) of rat liver synthesize and secrete fibronectin. Comparison with hepatocytes. J Hepatol 4:190-197

Roebroek AJ, Taylor NA, Louagie E, Pauli I, Smeijers L, Snellinx A, Lauwers A, Van de Ven WJ, Hartmann D, Creemers JW (2004) Limited redundancy of the proprotein convertase furin in mouse liver. J Biol Chem 279:53442-53450

Shiojiri N (1997) Development and differentiation of bile ducts in the mammalian liver. Microsc Res Tech 39:328-335

Shiojiri N, Inujima S, Ishikawa K, Terada K, Mori M (2001) Cell lineage analysis during liver development using the $\operatorname{spf}(\mathrm{ash})-$ heterozygous mouse. Lab Invest 81:17-25

Strick-Marchand H, Weiss MC (2003) Embryonic liver cells and permanent lines as models for hepatocyte and bile duct cell differentiation. Mech Dev 120:89-98

Suzuki A, Iwama A, Miyashita H, Nakauchi H, Taniguchi H (2003) Role for growth factors and extracellular matrix in controlling differentiation of prospectively isolated hepatic stem cells. Development 130:2513-2524

Tomizawa M, Toyama Y, Ito C, Toshimori K, Iwase K, Takiguchi M, Saisho H, Yokosuka O (2008) Hepatoblast-like cells enriched from mouse embryonic stem cells in medium without glucose, pyruvate, arginine, and tyrosine. Cell Tissue Res 333:17-27

Vassy J, Kraemer M, Chalumeau MT, Foucrier J (1988) Development of the fetal rat liver: ultrastructural and stereological study of hepatocytes. Cell Differ 24:9-24

Vassy J, Rigaut JP, Briane D, Kraemer M (1993) Confocal microscopy immunofluorescence localization of desmin and other intermediate filament proteins in fetal rat livers. Hepatology 17:293-300

Zhao R, Duncan SA (2005) Embryonic development of the liver. Hepatology 41:956-967 